- Almost all adults face the decision as to whether to have their third molars removed or not.

- Patients who were part of a prospective cohort study kept a diary of changes in their life quality each day for 7 days following third molar surgery and reported immense disturbances to their daily living

- Greater understanding of the outcomes of dental treatment from patients' perspectives can help to inform patients' treatment decision making processes and facilitate informed consent.

\title{
Changes in life quality following third molar surgery - the immediate postoperative period
}

\author{
C. McGrath, ${ }_{1}^{1}$ M. B. Comfort, ${ }_{1}^{2}$ E. C. M. Lo ${ }^{3}$ and Y. Luo ${ }^{4}$
}

Objectives This study describes patients' perceptions of changes in oral health related quality of life $(\mathrm{OHOOL})$ in the early postoperative period following third molar surgery.

Methods One hundred patients were enrolled in a prospective cohort study of the surgical removal of lower third molars under local anaesthetic. Two specific oral health related quality of life measures, $\mathrm{OHIP}-14$ and $\mathrm{OHOLLUK} \odot$ were administered to the study group prior to surgery. Standardized surgical and analgesic protocols were followed. Patients kept a diary of changes in life quality each postoperative day (POD) for 7 days, completing both OHIP-14 and OHQoLUK@ daily.

Results Both oral health related quality of life measures identified a significant deterioration in quality of life on POD1 $(P<0.01)$ and this remained evident on POD2 $(P<0.01)$, $P 0 D 3(P<0.01)$, $P O D 4(P<0.01)$ and POD5 $(P<0.05)$. By POD6 and POD7 there was no significant difference in quality of life compared with preoperative status $(P>0.05)$. Deterioration in life quality over the study period was associated with postoperative clinical findings $(P<0.05)$ : swelling and trismus.

Conclusion The study concludes that there is a significant deterioration in oral health related quality of life in the immediate postoperative period following third molar surgery; particularly during the first five days. This is associated with postoperative clinical findings. This has implication for patients deciding on third molar surgery and informed consent.

\section{INTRODUCTION}

Almost all young adults face the decision of whether to have their third molars extracted or not. ${ }^{1}$ The surgical removal of wisdom teeth under local anaesthesia is widely carried out in general dental practice and also occupies an appreciable amount of clinical time in many hospital oral and maxillofa-

$1^{1 *}$ Associate Professor ${ }^{2}$ Associate Professor ${ }^{3}$ Associate Professor ${ }^{4}$ Research Assistant, Faculty of Dentistry, University of Hong Kong, 34 Hospital Road, Hong Kong SAR, China. ${ }^{*}$ Correspondence to: Dr Colman McGrath, Faculty of Dentistry, University of Hong Kong, 34 Hospital Road, Hong Kong SAR, China.

E-mail:mcgrathc@hkucc.hku.hk cial surgery departments. ${ }^{2,3}$ The decision, however, to remove third molars is not straightforward and all too often misunderstandings about the possible outcomes of third molar surgery result in dentolegal negligence proceedings. ${ }^{4}$ The medico-legal defence societies have repeatedly reiterated the importance of communicating the potential risks of such procedures to patients to enable them to give their 'informed consent' for surgery. ${ }^{5,6}$ While a great body of evidence exists about the possible signs and symptoms following third molar surgery in terms of pain, swelling, trismus and parasthesia, surprisingly little is known about the consequences of these on a patient's life, and how it affects their day to day life or life quality. ${ }^{7-10}$

Assessment of the physical, social and psychological consequences of health states has been carried out for several decades now in medicine but only recently has it been employed in the dental arena. ${ }^{11}$ A plethora of oral health specific quality of life measures (questionnaires) have been developed and their psychometric properties evaluated. ${ }^{12}$ Their value and use in an oral and maxillofacial surgery setting are potentially manifold: not only in providing information about the importance of oral health status and how treatments may affect life quality; they may also emerge as important tools to measure quality, effectiveness and efficiency of treatment approaches. ${ }^{13}$

\section{AIMS}

The aims of this study were firstly, to assess the impact of oral health on the quality of life of patients awaiting third molar surgery; secondly, to measure patients' perceptions of changes in their life quality in the immediate postoperative period for 7 days; and thirdly, to identify factors associated with changes in life quality.

\section{METHODS \\ Sample}

A consecutive sample of 100 patients awaiting lower third molar surgery at the Oral and Maxillofacial unit of the University of Hong Kong was recruited to participate in this study. Local ethics approval was obtained and written consent was obtained from all patients. Surgery was performed on an outpatient basis under local anesthesia following the standard surgical and anaesthetic protocols used at the teaching hospi- 


\section{RESEARCH}

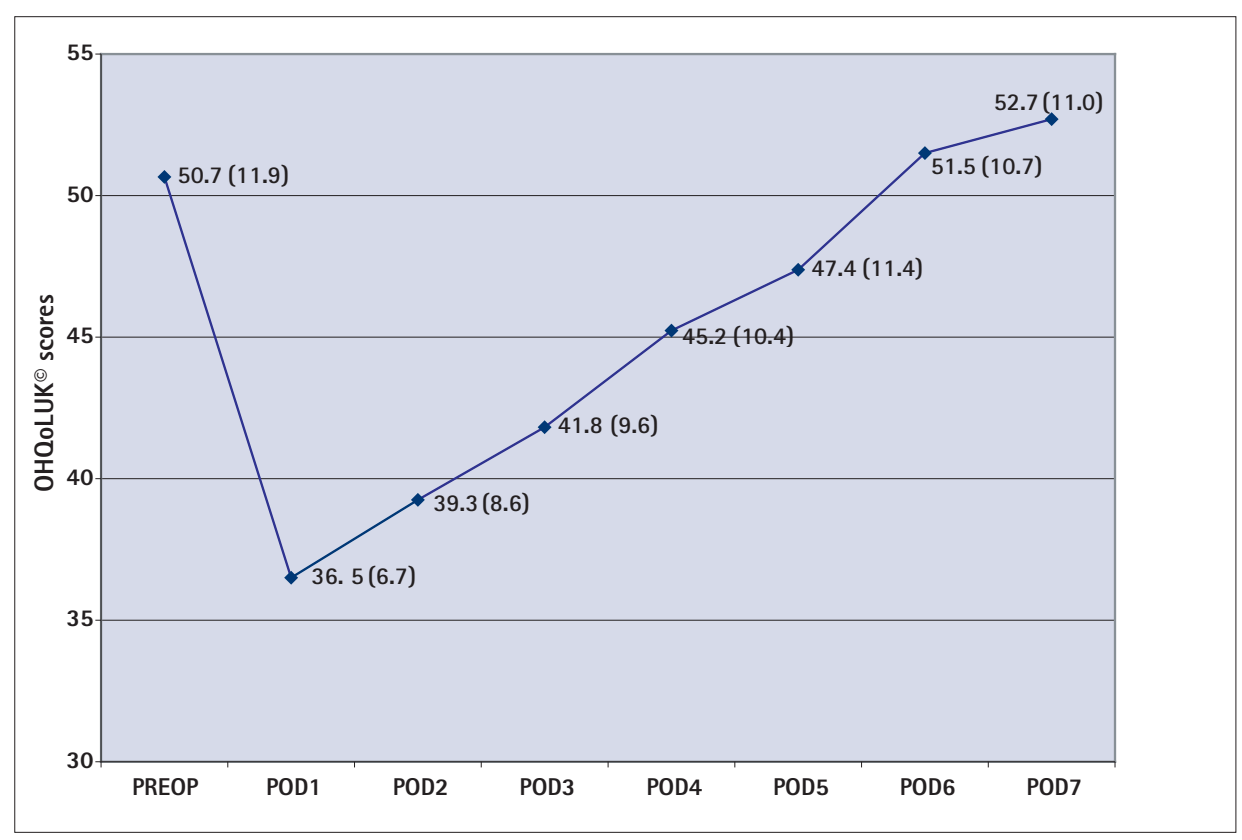

Figure 1 Mean OHQoLUK scores (95\%

confidence intervals) over the study period)

tal. ${ }^{14 *}$ Standard postoperative instructions were given and standard analgesics were prescribed ${ }^{\dagger}$ for all patients. A postoperative appointment was scheduled for one week following the surgery for removal of sutures.

\section{Data collection}

Preoperatively (on the day of surgery) the study participants underwent an oral examination and were asked to complete a questionnaire. The self-completed questionnaire incorporated the two patient-centred outcome measures; the 14-item oral health impact profile (OHIP-14) ${ }^{15}$ and the 16 -item UK oral health related quality of life measure (OHQoLUK $\left.{ }^{\odot}\right){ }^{16}$ These measures are based on two conceptually distinct models of oral health, the former based on the World Health Organization (WHO) model of 'disease-impairmentdisability-handicap'17 and the latter on an updated WHO model of 'structure-function-ability-participation' which incorporates both negative and positive influences of health. ${ }^{18}$

Details of the surgery were recorded and postoperatively patients were presented with a 'recovery log diary' and asked to complete the questionnaire daily for 7 days. Participants were given a contact telephone number should any questions about the study arise. On the seventh day postoperatively patients underwent an oral examination for signs and symptoms of surgical sequelae. Patients were asked about pain and paraesthesia. Presence of bruising and swelling were recorded, and evidence of trismus was assessed by measuring range of opening (in $\mathrm{mm}$ ) by the surgeon in charge.

\section{Data analysis}

Scores were derived from both questionnaires by summating the responses to each of the individual questions within the measures. For OHIP-14, each item was scored: 'never' - score 0, 'hardly ever' - score 1, 'occasionally' - score 2, 'fairly often' - score 3, 'very often' - score 4. Possible OHIP-14 scores range from 0 to 56 . A high OHIP score represents a poor quality of life. For the OHQoLUK ${ }^{\odot}$ the response categories were: 'very bad effect' - score 1, 'bad effect' - score 2, 'no effect' - score 3, 'good effect' - score

*Xylocaine ${ }^{\circledR} 2 \%$ with 1:80,000 adrenaline was administered. Buccal flaps were used, lingual tissues were retracted and protected, buccal and distal bone was removed with burs, tooth sectioning was done with burs where necessary, and sutures were placed to achieve primary or secondary closure as appropriate.

${ }^{\dagger} 12$ panadeine ${ }^{\circledR}$ tablets (paracetamol $500 \mathrm{mg}$ and codeine $8 \mathrm{mg}$ ) and patients were instructed to take 1-2 tablets 4-6 hourly as required.
4, 'very good effect' - score 5. Possible OHQoLUK ${ }^{\odot}$ scores range from 16 to 80 . A low $\mathrm{OHQ}_{\mathrm{LLUK}}{ }^{\odot}$ scores represents a poor quality of life.

Mean OHIP-14 and OHQoLUK ${ }^{\odot}$ scores were plotted to display patients' perceptions of their life quality over the immediate postoperative study period (7-day period). Variations in oral health related quality of life (OHIP-14 and OHQoLUK ${ }^{\odot}$ ) scores between preoperative and postoperatively states were assessed employing paired sample $t$ tests, with the Bonferroni correction method for multiple tests. Simple frequency distributions were computed for each item, and descriptive statistics were used to describe patient responses to all items over the study period. Associations between postoperative clinical findings and changes in oral health related quality of life were explored employing Chi-square statistics.

\section{RESULTS}

Seven subjects failed to complete the initial 7-postoperative day (POD) study, citing time and other constraints as reasons why they were unable to complete the recovery log diary or attend for postoperative examination, and were excluded from the analysis. Thus, 93 out of the 100 subjects' data were available for analysis. The mean age of the study subjects was 26 years (sd 8) and the median age was 24 years. There were 67 women (76\%) and 26 men (28\%). All were of Chinese ethnicity. One mandibular third molar was surgically removed in each case; 55\% (51) were lower left and 45\% (42) lower right third molars. In most cases, an erupted upper third molar (on the same side as the lower third molar) was also removed by forceps extraction: upper right $(32 \%, 30)$ or upper left $(25 \%, 23)$.

In the immediate postoperative period following surgery there was a significant decrease in quality of life among the group compared with preoperative status. There was a dramatic reduction in the mean $\mathrm{OHQ}_{\mathrm{LLUK}}{ }^{\odot}$ score of the subjects on POD 1 following surgery (mean difference 14.16, 95\%CI 11.66, 16.65; $\mathrm{P}<0.001$ ), and this remained evident on POD 2 (mean difference 11.41 , 95\%CI 8.67, 14.15; $\mathrm{P}<0.001$ ), POD 3 (mean difference 8.84, 95\%CI 6.04, 11.64; $\mathrm{P}<0.001$ ), POD 4 (mean difference 5.43, 95\%CI 2.42, 8.44; $\mathrm{P}<0.001$ ) and POD 5 (mean difference 3.28, 95\%CI 0.19, 6.38; $\mathrm{P}=0.04)$, as shown in Figure 1. Similarly there were large changes in OHIP-14 scores during the postoperative period compared with preoperative status (Figure 2). There was an increase in the mean OHIP-14 score of the subjects (increase burden on life quality) on POD 1 following surgery and this remained statistically significant for five days: POD 1 (mean difference 21.01, 95\%CI 18.85, 23.18; $\mathrm{P}<0.001$ ), POD 2 (mean difference 15.51, 95\%CI 12.91, 18.01; 


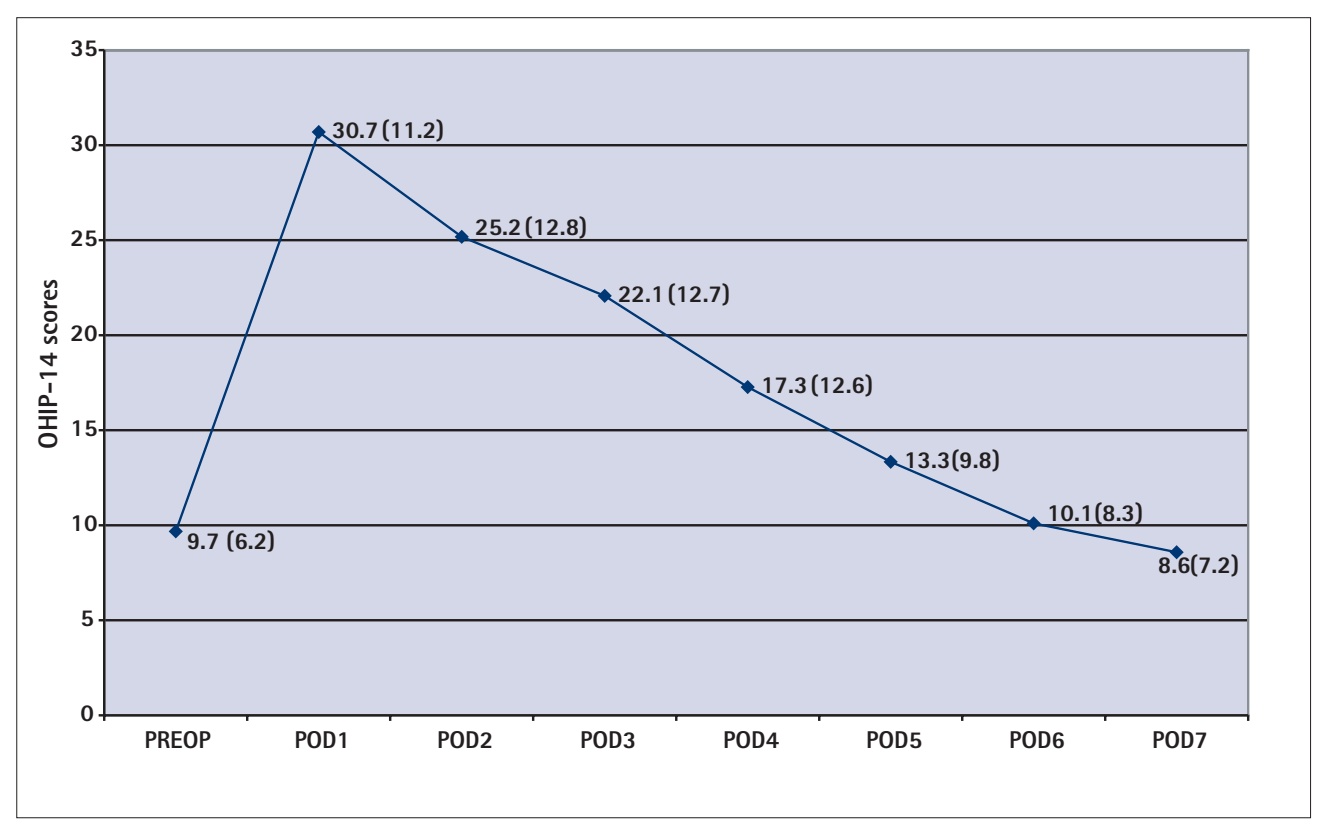

Figure 2 Mean OHIP-14 values (95\% confidence intervals) over the study period

$\mathrm{P}<0.001$ ), POD 3 (mean difference 12.39, 95\%CI 9.86, 14.91; $\mathrm{P}<0.001$ ), POD 4 (mean difference 7.59, 95\%CI 5.10, 10.13; $\mathrm{P}<0.001$ ) and POD 5 (mean difference 3.67, 95\%CI 1.68, 5.66; $\mathrm{P}<0.001$ ) compared with preoperative status. On POD 6 and POD 7, the mean OHIP-14 and $0 \mathrm{HQ}_{\mathrm{OLUK}}{ }^{\odot}$ scores approximated preoperative values $(\mathrm{P}>0.05)$. There was no significant differences in changes in mean OHIP-14 score or mean OHQoLUK ${ }^{\odot}$ scores, between those who had an upper third molar removed at the same time as having their lower third molar removed compared with those who had only their lower third molars removed during the surgery $(\mathrm{P}>0.05)$.

Specifically, when the responses to the 16 individual items of the $\mathrm{OHQOLUK}^{\odot}$ measure over the study period were examined, deterio-

\begin{tabular}{|c|c|c|}
\hline (number) & & $\begin{array}{l}\text { Percentage } \\
\text { of subjects }\end{array}$ \\
\hline Symptom level & $\begin{array}{l}\text { Comfort } \\
\text { Breath odour }\end{array}$ & $\begin{array}{l}81 \%(75) \\
58 \%(54)\end{array}$ \\
\hline Body function level & $\begin{array}{l}\text { Eating } \\
\text { Appearance } \\
\text { General health } \\
\text { Speech } \\
\text { Smiling/ laughing }\end{array}$ & $\begin{array}{l}89 \%(83) \\
76 \%(71) \\
61 \%(57) \\
84 \%(78) \\
70 \%(65)\end{array}$ \\
\hline At person level & $\begin{array}{l}\text { Relax/sleep } \\
\text { Confidence } \\
\text { Mood } \\
\text { Carefree manner } \\
\text { Personality }\end{array}$ & $\begin{array}{l}67 \%(62) \\
50 \%(46) \\
59 \%(55) \\
54 \%(50) \\
36 \%(33)\end{array}$ \\
\hline At social level & $\begin{array}{l}\text { Social life } \\
\text { Romantic relationships } \\
\text { Work/ usual jobs } \\
\text { Finance }\end{array}$ & $\begin{array}{l}73 \%(68) \\
40 \%(37) \\
67 \%(62) \\
19 \%(18)\end{array}$ \\
\hline
\end{tabular}

ration in life quality was observed across a broad range of aspects; having influences on symptoms, at a body level, at a personal level and on a social level during the immediate postoperative period (Table 1). Likewise, when changes at the domain level of the OHIP14 measures over the study period were explored, major changes in oral health impact were observed (Table 2). Immense increases (over 75\%) in functional limitation, physical pain, psychological discomfort, physical disability, psychological disability, social disability and handicap of the group were apparent.

At the postoperative review, seven days following surgery, few subjects had clinical signs of postoperative sequelae aside from trismus $(27 \%, 25)$ and intra-oral or extra-oral swelling $(25 \%, 23)$. One subject had signs of dysaesthesia and five had signs of intra-oral or extra-oral bruising. However, many still perceived that their oral health was detracting from their quality of life to a greater extent than prior to the surgery. Forty-two percent (39) had lower OHQoLUK ${ }^{\odot}$ scores and 27\% (25) had higher 0HIP-14 scores. This was associated with postoperative clinical findings. Those with signs of a swelling were more frequently experiencing reduced oral health related quality of life at the end of the study period compared with those without signs of swelling intra-orally or extra-orally $(\mathrm{P}<0.05)$, Table 3 . Presence of swelling was associated with over a three and a half times the odds of having a reduced OHQoLUK ${ }^{\odot}$ score $(\mathrm{OR}=3.59,95 \% \mathrm{CI}$

Table 3 Distribution of subjects according to the change in their quality of life and postoperative clinical findings, 7 days after surgery.

\begin{tabular}{|c|c|c|c|c|c|}
\hline & & \multicolumn{2}{|c|}{ Reduced $\mathrm{OHOoLUK}^{\odot}$ score } & \multicolumn{2}{|c|}{ Increased OHIP-14 score } \\
\hline & & Yes & No & Yes & No \\
\hline \multirow[t]{2}{*}{ Swelling present* } & Yes & 15 & 08 & 10 & 13 \\
\hline & No & 24 & 46 & 15 & 53 \\
\hline \multirow[t]{2}{*}{ Trismus* } & Yes & 16 & 09 & 12 & 13 \\
\hline & No & 23 & 45 & 13 & 55 \\
\hline
\end{tabular}

Table 2 Increase in oral health impact on life quality over the study period (OHIP domains adversely affected).

Functional limitations

Physical pain

Psychological discomfort

Physical disability

Psychological disability

Social disability

Handicap
Had trouble pronouncing words or felt their sense of taste has worsened Had a painful aching in their mouth or found it uncomfortable to eat any foods Have been self-conscious or felt tense

Had an unsatisfactory diet or had to interrupt meals

Found it difficult to relax or have been a bit embarrassed

Have been irritable with other people or had difficulty doing usual jobs

Felt life in general was less satisfying or have been totally unable to function
Percentage (number) of subjects

$89 \%(83)$

$90 \%(84)$

$81 \%(75)$

$88 \%(82)$

$79 \%(74)$

$83 \%(77)$

$81 \%(76)$ 


\section{RESEARCH}

$1.21,11.13 ; \mathrm{P}=0.009)$ and over two and a half times the odds ratio of having an increased OHIP-14 score $(\mathrm{OR}=2.72$, 95\% CI 0.87 , 8.26; $\mathrm{P}=0.047)$. Furthermore, those who had evidence of trismus, having limited mouth opening of less than $30 \mathrm{~mm}$, more frequently had reduced oral health related quality of life compared with those without such trismus $(\mathrm{P}<0.01)$. Presence of trismus was associated with approximately a three and a half times the odds of having a reduced OHQoLUK $^{\odot}$ score $(\mathrm{OR}=3.48,95 \% \mathrm{CI}$ $1.21,10.29 ; \mathrm{P}=0.009)$ and almost four times the odds ratio of having an increased OHIP-14 score (OR=3.91, 95\%CI 1.28, 11.74; $\mathrm{P}=0.005)$.

\section{DISCUSSION}

The study group is limited by the relatively small and non-random nature of the sample and this affects the generalizability of the findings. That aside, the sample does offer an insight into the experiences of young and healthy adults following third molar surgery under local anaesthetic, and in that way offers an insight into what is likely to occur in the 'best scenario' and also into cases likely to be treated in general dental practice.

Both measures identified a significant deterioration in oral health related quality of life in the immediate postoperative study period, notably for the first five days. This is important information that may be useful for improving the decision making process for patients because it quantifies for patients and surgeons the actual experiences of a group of patients using lay terminology.

In addition, both measures demonstrated deterioration in life quality across a broad range of domains, expanding our understanding of the impact of third molar surgery beyond signs and symptoms, providing a more comprehensive assessment of physical, social and psychological consequences that affect patients at a body, personal and societal level. Such information may help create realistic expectations for similar surgical candidates considering third molar surgery and in that way will enhance the informed consent procedures. ${ }^{19}$

Of note, while both measures illustrated a sharp reduction in oral health related quality of life immediately followed surgery (on day 1), there was however a steady increase in recovery from there on. By day 7 postoperatively, mean OHQoLUK ${ }^{\odot}$ and OHIP14 scores approximated preoperative values. As to whether this trend of recovery or improvement in life quality continues and does so significantly over time warrants further research. Employing patient's perceptions of outcomes from oral surgery is important in establishing the value of third molar surgery. Sometimes 'cure' is worse that 'disease', in terms of impact on life quality, and it is important to identify such scenarios to inform polices and guidelines and in promoting evidence based practice. ${ }^{20}$

At the end of the study period, many patients were still experiencing a reduction in their oral health related quality of life compared with preoperative status. This was associated with postoperative clinical findings. It was the experience of trismus, limitation of mouth opening less than $30 \mathrm{~mm}$, and clinical evidence of swelling (intra-orally or extra-orally) that was associated with reduced oral health related quality of life at the end of the study period. The limitation of mouth opening is likely to be a particularly troubling experience perceived by patients following third molar surgery. ${ }^{21}$ Similarly, swelling is likely to have influences of comfort, function and aesthetics. Identifying factors and best treatment approaches to limit or avoid trismus and swelling is important in improving patients' recovery and in reducing the burden that third molar surgery places on life quality in the immediate postoperative period.

\section{CONCLUSION}

The study concludes that there is a significant deterioration in oral health related quality of life in the immediate postoperative period following third molar surgery, which slowly returns to a preoperative level in 6 to 7 days. This deterioration in life quality is associated with postoperative findings (trismus and swelling). These findings have implications in understanding patient's perceptions of changes in life quality following third molar surgery and in informing patients and surgeons in the treatment decision making process.

1. Song F, Landes D P, Glenny A M, Sheldon T A. Prophylactic removal of impacted third molars: an assessment of published reviews. Br Dent J 1997; 182: 339-346.

2. Thomas $D$, Walker $R$, Smith $A$, Shepherd J. The provision of oral surgery services in England and Wales 1984-1991. Br Dent J 1994; 176: 215-219.

3. Worrall S F, Riden K, Haskell R, Corrigan A M. UK National Third Molar project: the initial report. Br J Oral Maxillofac Surg 1998; 36: 14-18.

4. Moles D R, Simper R D, Bedi R. Dental negligence: a study of cases assessed at one specialised advisory practice. Br Dent J 1998; 184: 130-133.

5. Williams M. Post-operative nerve damage and the removal of the mandibular third molar: a matter of common consent. Br J Oral Maxillofac Surg 1996; 34: 386-388.

6. Layton S A. Informed consent in oral and maxillofacial surgery: a study of its efficacy. Br J Oral Maxillofac Surg 1992; 30: 319-322.

7. Seymour R A, Walton J G. Pain control after third molar surgery. Int J Oral Surg 1984: 13: 457-385.

8. Lopes V, Mumenya R, Feinmann C, Harris M. Third molar surgery: an audit of the indications for surgery, post-operative complaints and patient satisfaction. Br J Oral Maxillofac Surg 1995; 33: 33-35.

9. Mercier P, Precious D. Risks and benefits of removal of impacted third molars. A critical review of the literature. Int J Oral Maxillofac Surg 1992; 21:17-27.

10. Brann C R, Brickley M R, Shepherd J P. Factors influencing nerve damage during lower third molar surgery. Br Dent J 1999; 186: 514-516.

11. McGrath C, Bedi R. A review of the influences of oral health on the quality of life. Int J of Health Ed 1999; 37: 116-119.

12. Slade G D, ed. Measuring Oral Health and Quality of Life. Chapel Hill: University of North Carolina, Dental Ecology, 1997.

13. McGrath C, Bedi R. The value and use of 'quality of life' measures in the primary dental care setting. Primary Dent Care 1999; 6: 53-57.

14. Dimitroulis G. A synopsis of minor oral surgery. Oxford: Wright, 1997.

15. Slade G D. Derivation and validation of a short-form oral health impact profile. Community Dent Oral Epidemiol 1997; 25: 284-290.

16. McGrath C, Bedi R. An evaluation of a new measure of oral health related quality of life - OHQoLUK ${ }^{\odot}$. Community Dent Health 2001; 18: 138-143.

17. World Health Organization. International Classification of Impairments, Disability and Handicaps. Geneva: WHO, 1980

18. World Health Organization. ICIDH-2 International Classification of Functioning, Disability and Health. Geneva: WHO, 1998.

19. Ogden G R, Bissias E, Ruta D A, Ogston S. Quality of life following third molar removal: a patient versus professional perspective. Br Dent J 1998; 185: 407-410.

20. Goodey R D, Brickley M R, Armstrong R A, Shepherd J P. The minor oral surgery outcome scale: a multi-attribute patient-derived outcome measure. J Oral Maxillofac Surg 2000; 58: 1096-1101.

21. Lopes $\mathrm{V}$, Mumenya R, Feinmann $\mathrm{C}$, Harris M. Third molar surgery: an audit of the indications for surgery, post-operative complaints and patient satisfaction. Br J Oral Maxillofac Surg 1995; 33:33-35.

This study was supported by a research grant (CRCG) from the University of Hong Kong, Hong Kong SAR, CHINA. 